\title{
Influence of Total Knee Arthroplasty on Hip Rotational Range of Motion
}

\author{
Tatsunori Kataoka, Yasushi Oshima, Norishige Iizawa, \\ Tokifumi Majima and Shinro Takai \\ Department of Orthopaedic Surgery, Nippon Medical School, Tokyo, Japan
}

\begin{abstract}
Background: Total knee arthroplasty (TKA) aims to correct the rotation, as well as the alignment and articulation, of the osteoarthritic knee. We hypothesized that, in addition to improving knee kinematics, TKA affects hip rotational movement. The objective of this study was to evaluate variation in lower extremity alignment and hip rotational range of motion (ROM) after TKA.
\end{abstract}

Methods: A total of 47 patients (53 knees) with primary varus knee osteoarthritis who were scheduled for primary TKA at our center were enrolled. Hip rotational ROM was measured with the patient in supine position with $90^{\circ}$ flexion of the hip and knee before and 3 weeks after TKA. Plain radiography and computed tomography were used to compare variations in tibial axis alignment and femoral axis alignment after bone resection, which was defined as changes in the joint lines of the distal femur and the proximal tibia.

Results: Average internal ROM, and the sum of internal and external hip rotational ROM, increased significantly; however, external hip rotational ROM did not significantly differ after TKA. Imaging findings showed that the axis of the lower leg externally rotated by $2.5^{\circ}$, with a $4^{\circ}$ internal rotation of the distal femur and a $6.5^{\circ}$ correction of the varus deformity.

Conclusion: TKA changed the neutral position of hip rotational movement and increased hip rotational ROM. (J Nippon Med Sch 2020; 87: 191-196)

Key words: knee osteoarthritis, total knee arthroplasty, hip rotational movement

\section{Introduction}

Osteoarthritis of the knee (knee OA) is a common disease in middle-aged and older adults; knee OA was detected radiographically in $62 \%$ of Japanese older than 60 years ${ }^{1}$. Moreover, 33\% of people have knee pain due to OA, which diminishes their ability to perform activities of daily living (ADL) and limits knee range of motion $(\mathrm{ROM})^{2,3}$. Conservative treatments involving medications and rehabilitation are applied for early knee OA; for advanced knee OA, total knee arthroplasty (TKA) is performed to eliminate pain, increase $\mathrm{ADL}$, and improve quality of life ${ }^{4,5}$. TKA aims to correct the malalignment and malrotation of lower extremities seen in advanced knee OA, and to improve knee articulation.

A standard procedure for evaluating hip rotational $\mathrm{ROM}$ is to measure movement of the lower leg from the vertical line, ie, the imaginary line extending from the center of the patella parallel to the axis of the body trunk when the patient is in supine position with $90^{\circ}$ flexion of the hip and $\mathrm{knee}^{6,7}$. In healthy knees, the lower leg is anatomically positioned on this vertical line. However, varus knee deformity may cause the lower leg axis to rotate inward from the vertical line. Although this inward position of the lower leg is assumed to be the neutral position of hip rotational movement, rotational ROM is measured after internally rotating the hip and placing the lower leg on the vertical line. Therefore, internal rotational ROM is believed to be underestimated and external rotational ROM overestimated when the measurement is performed from the vertical line using the standard procedure. Because this malalignment is corrected in $\mathrm{TKA}^{8}$, the position of the lower leg in the supine position with $90^{\circ}$ flexion of the hip and knee is expected to be realigned to the normal position. We hypothesized

Correspondence to Yasushi Oshima, MD, PhD, Department of Orthopaedic Surgery, Nippon Medical School, 1-1-5 Sendagi,

Bunkyo-ku, Tokyo 113-8603, Japan

E-mail: y-oshima@nms.ac.jp

https://doi.org/10.1272/jnms.JNMS.2020_87-401

Journal Website (https://www.nms.ac.jp/sh/jnms/) 
(1)

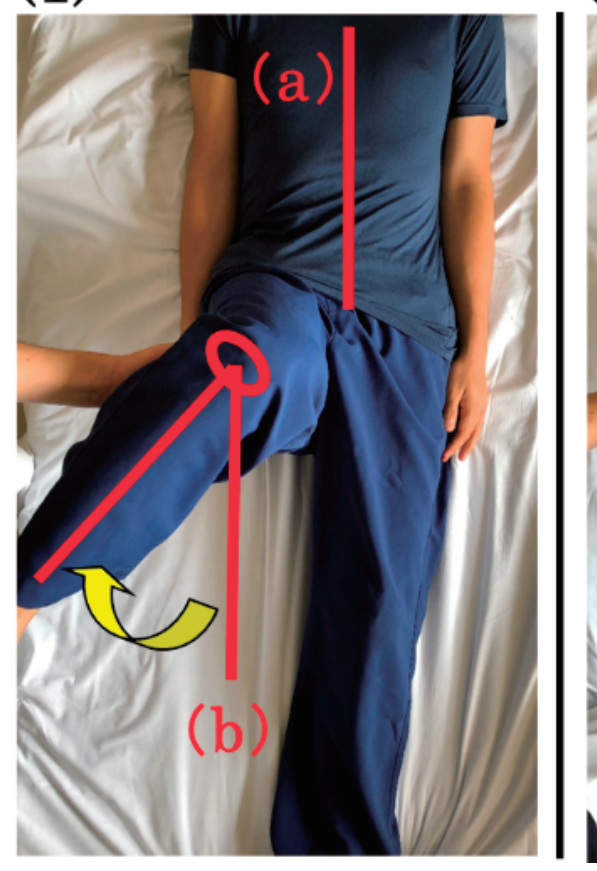

(2)

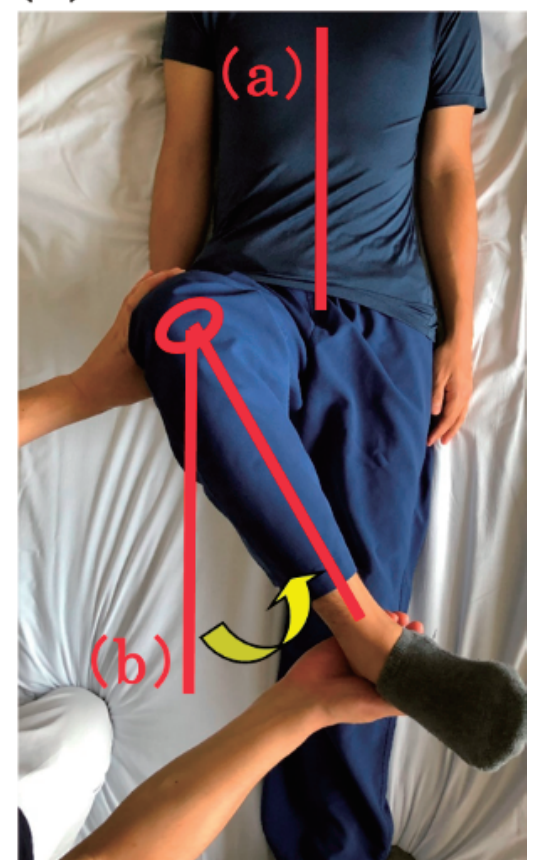

Fig. 1 Hip rotational range of motion is evaluated in the supine position with $90^{\circ}$ flexion of the hip and knee. Internal (1) and external (2) rotational range of motion (arrows), (a) axis of the body trunk, and (b) vertical line from the patella parallel to (a).

that the ratio of internal and external rotation varies after bone resections in TKA. The objective of this study was to evaluate variation in hip rotational ROM after TKA.

\section{Patients and Methods}

This study was approved by the relevant institutional review board (R1-05-1133), and all patients provided informed consent for participation. The inclusion criterion for this study was severe varus knee OA patients scheduled for primary TKA in our center between July 2017 and June 2018. The hip-knee-ankle (HKA) angle is the angle between the mechanical axes of the femur and tibia, and varus knee was defined as an HKA angle less than $-3^{\circ 9,10}$. Patients with knee flexion less than $90^{\circ}$; valgus knee deformity; secondary knee OA due to rheumatoid arthritis, infection, or trauma; hip OA; or past surgery involving the lower extremities were excluded. A total of 47 patients were enrolled, and 6 underwent simultaneous bilateral TKA. These patients were evaluated separately, and the total number of knees was 53 (8 knees were from 7 men and 45 knees from 40 women). Average patient age of all cases was 76.5 \pm 5.8 years (range, 64-87).

\section{Knee ROM and HKA Angle}

Knee ROM was measured with the patient in supine position, and HKA angle was calculated by using antero- posterior whole-leg standing plain radiographs before and 3 weeks after TKA.

\section{Hip Rotational ROM}

Hip rotational ROM was measured in supine position with $90^{\circ}$ flexion of the hip and knee. One evaluator passively rotated the patient's hip internally and externally, and the other evaluator measured the angle by using a goniometer with the stable arm parallel to the trunk and the mobile arm on the lower leg axis (Fig. 1).

Alignment Change of the Lower Extremity after TKA

In $90^{\circ}$ flexion of the hip and knee, the position of the lower leg was determined by examining the relationship between the posterior condylar line (PCL) of the femur and the joint line of the proximal tibia. Rotation of the distal femur in the axial plane was measured as the posterior condylar angle (PCA), which is the angle between the PCL and surgical epicondylar axis (SEA) on plain computed tomography (CT). The PCL was defined as the line connecting the medial and lateral posterior condyles of the femur preoperatively and as the line connecting the medial and lateral posterior condyles of the femoral implant after TKA. The SEA is the line connecting the sulcus of the medial epicondyle and the prominence of the lateral epicondyle of the femur. When the PCL was externally rotated, as compared with the SEA, the PCA 
(1)

(2)

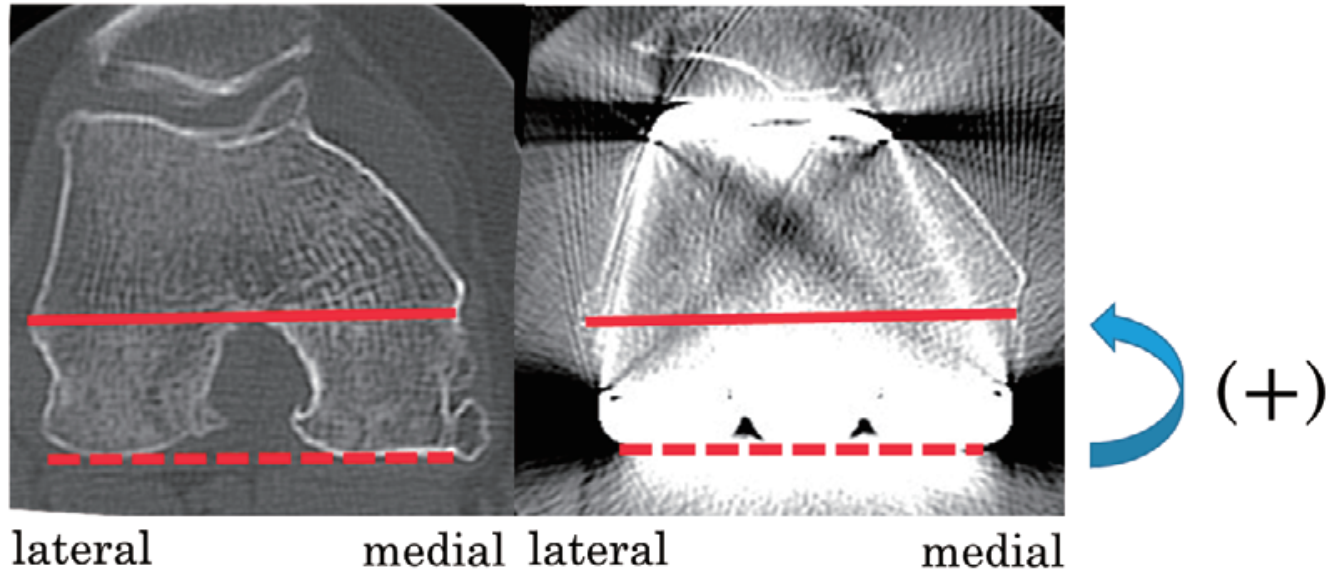

Fig. 2 The posterior condylar angle is measured using plain computed tomography before (1) and after (2) total knee arthroplasty. The solid line represents the surgical epicondylar axis, and the dotted line represents the posterior condylar line. This shows $2.9^{\circ}$ negative in (1) and $0^{\circ}$ in (2).

(1)

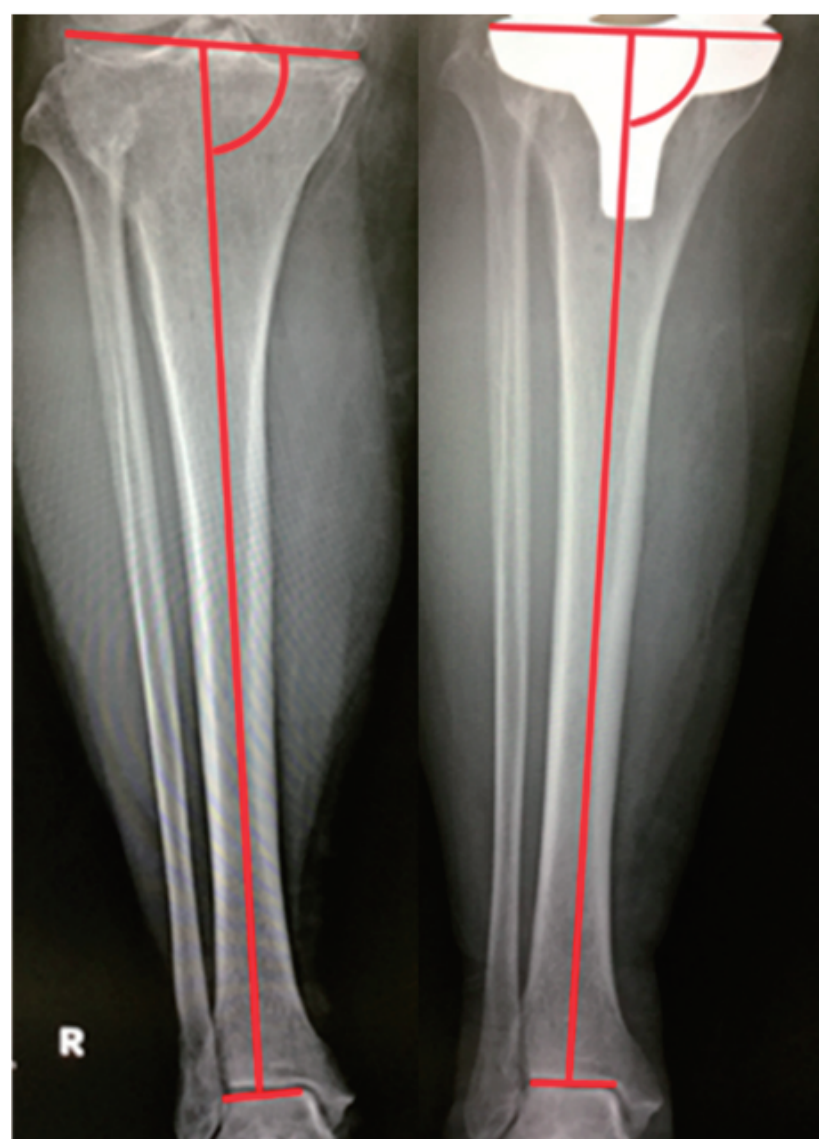

Fig. 3 The medial proximal tibial angle is measured using plain radiography before (1) and after (2) total knee arthroplasty. This shows $83^{\circ}$ in (1) and $90^{\circ}$ in (2). was given a positive value (Fig. 2).

Alignment of the proximal tibia on the coronal plane was measured as the medial proximal tibial angle (MPTA), which is the medial angle between the joint line of the proximal tibia and the mechanical axis of the tibia on plain anteroposterior radiographs (Fig. 3). The PCA and MPTA were measured before and 3 weeks after TKA, and variation in lower leg position, ie, the sum of the variation of the PCA and MPTA (a positive value indicates external change of the PCL and tibial joint line), was calculated.

\section{Femoral Anteversion}

Femoral anteversion was measured by using CT according to the method described by Hernandez ${ }^{11}$. The line from the center of the femoral head to the center of the femoral neck was detected on a single slice, and the PCL was determined on another slice. Thereafter, these 2 slices were superimposed, and the angle between the lines was calculated (Fig. 4). Femoral anteversion was given a positive value, and retroversion was given a negative value.

\section{Statistical Analysis}

All values are presented as means \pm SD. All data were analyzed by using the SPSS version 25.0 statistical software (IBM Corp., Armonk, NY, USA). Variation in hip rotational ROM was compared with the two-tailed t-test. A $p$-value of $<0.05$ was considered to indicate statistically significance. 


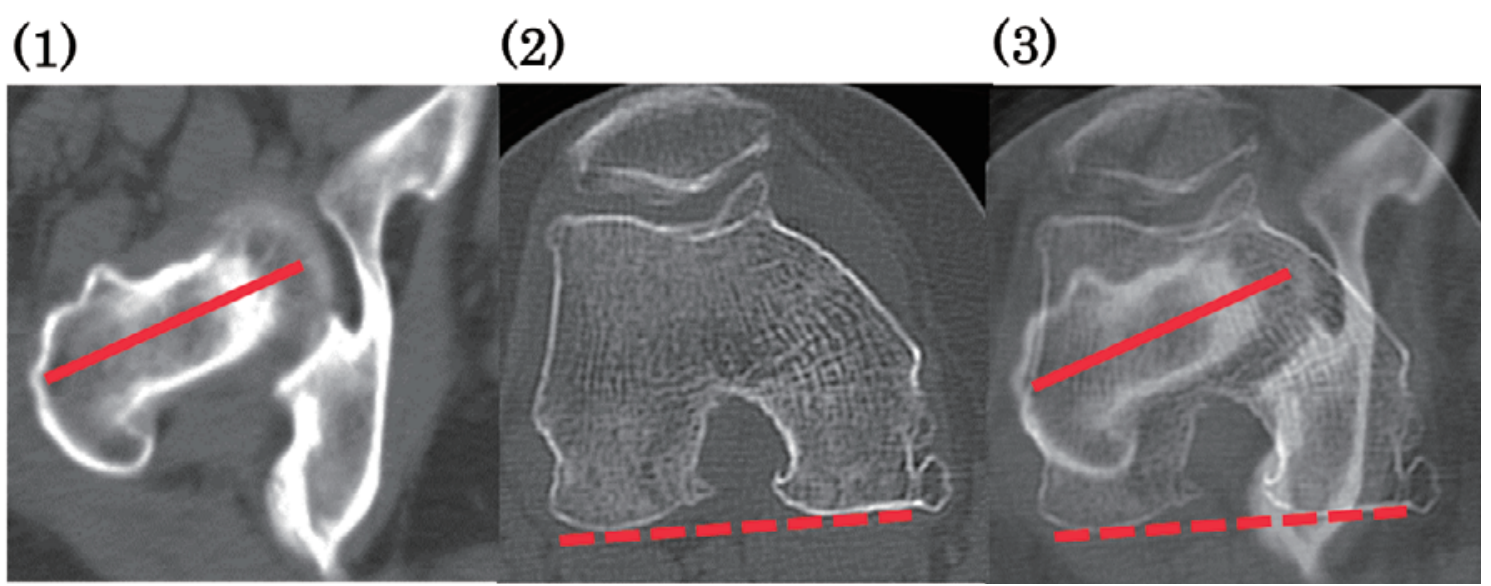

Fig. 4 Femoral anteversion was measured using plain computed tomography. The femoral neck is the line connecting the center of the femoral head and the center of the femoral neck (1). The posterior condylar line is the line connecting the medial and lateral posterior condyles of the femur (2). Two slices were superimposed, and the angle between the two lines was measured (3).

Table 1 Knee characteristics before and after total knee arthroplasty

\begin{tabular}{lccc}
\hline & Before TKA & After TKA & $p$-value \\
\hline Knee extension & $-7.2 \pm 7.9^{\circ}$ & $-1.5 \pm 3.7^{\circ}$ & $<0.0001$ \\
Knee flexion & $121.6 \pm 15.1^{\circ}$ & $118.8 \pm 10.3^{\circ}$ & 0.13 \\
Internal hip rotation & $22.3 \pm 11.2^{\circ}$ & $28.1 \pm 10.5^{\circ}$ & 0.0001 \\
External hip rotation & $37.9 \pm 10.6^{\circ}$ & $38.2 \pm 9.7^{\circ}$ & 0.82 \\
Internal + External hip rotation & $60.5 \pm 15.4^{\circ}$ & $66.2 \pm 12.0^{\circ}$ & 0.004 \\
HKA angle & $-12.1 \pm 5.0^{\circ}$ & $-1.0 \pm 3.5^{\circ}$ & $<0.0001$ \\
PCA & $-3.4 \pm 1.8^{\circ}$ & $0.6 \pm 2.2^{\circ}$ & $<0.0001$ \\
MPTA & $83.5 \pm 2.9^{\circ}$ & $90.0 \pm 2.3^{\circ}$ & $<0.0001$ \\
Femoral anteversion & $5.9 \pm 7.8^{\circ}$ & $2.8 \pm 7.3^{\circ}$ & $<0.0001$ \\
\hline
\end{tabular}

Abbreviations: Hip-knee-ankle, HKA; posterior condylar angle, PCA; medial proximal tibial angle, MPTA; total knee arthroplasty, TKA.

Knee extension, internal and total hip rotational ROM, PCA and MPTA were significantly improved after TKA $(p<0.05)$. Femoral anteversion decreased significantly $(p<0.05)$.

\section{Results}

Knee extension was significantly improved $(p<0.05)$ after TKA. Although knee flexion decreased, the change was not statistically significant (Table 1). The average internal ROM and sum of the internal and external hip rotational ROM (total rotational ROM) increased significantly ( $p<$ 0.05). However, there was no significant difference in external hip rotational ROM after TKA (Table 1). After bone resection, HKA angle, PCA, MPTA and femoral anteversion had significantly changed $(p<0.05)$. The mean axis of the lower leg was thus externally rotated by $2.5 \pm$ $4.3^{\circ}$ after TKA.

\section{Discussion}

Because TKA corrects the rotation, as well as alignment and articulation of the knee, we evaluated the influence of TKA on hip rotational movement. Although internal hip rotational ROM increased, external hip rotational ROM did not decrease.

Because the knee rotation differed after TKA, we calculated PCA and femoral anteversion. The posterior condyle is cut parallel to the SEA in the measured resection technique; thus, PCA increases to $0^{\circ}$ after resection. This process also decreases femoral anteversion. The extent of the variations in PCA and femoral anteversion is theoretically the same, which was confirmed in our results. Thus, we discuss only PCA in our examination of femoral rotational alignment.

In the medial OA knee, the lower leg axis moves inward in persons with varus knee deformity. TKA aims to improve this malalignment by shifting the lower leg axis outward, with variation of the PCA and MPTA. The neu- 
tral position of the lower leg becomes almost parallel to the body trunk in the supine position, with $90^{\circ}$ flexion of the hip and knee post-TKA. Therefore, the ratio of internal and external rotational ROM theoretically varies. We believe that this increased internal rotational ROM and decreased external rotational ROM. The neutral position of the lower leg was calculated to be externally rotated, and internal rotational ROM was increased; however, the external rotational ROM was not decreased.

In normal knees, the lateral tibiofemoral joint gap is physiologically larger than the medial gap ${ }^{12}$. Consequently, there has been a trend toward more-limited release of medial soft tissues, as compared with conventional TKA, and the creation of a rectangular gap preserves natural knee kinematics ${ }^{13}$. In recent TKAs, in addition to bone resections, the medial osteophyte is removed to improve the varus knee deformity and relax the medial soft tissue, and the additional process of minimal medial soft tissue release is used to adjust the mediallateral joint gap. Although bone resections and intraoperative soft tissue releases likely affect hip rotational ROM, even in the short term, only the influence of bone resection was evaluated in this study. Future studies should investigate the effect of osteophyte removal and joint gaps.

Hip rotational ROM differed after TKA in our patients. Thus, TKA might have long-term effects on the biomechanical properties of soft tissues around the hip joint ${ }^{14}$, including the capsular ligaments and the muscles of the lower extremities. During $90^{\circ}$ flexion of the hip and knee, the ischiofemoral and pubofemoral ligaments are the primary restraints for internal hip rotational movement, and the iliofemoral ligament is the primary restraint for external hip rotational movement ${ }^{15-17}$. Thus, long-term followup is necessary in order to evaluate the effects of TKA on these ligaments and muscles and the further influence on hip rotational movement.

The ranges of internal and external hip rotational ROM were reported to be similar- $43.5^{\circ}$ and $42.2^{\circ}$, respectivelyin a population of healthy young Asians ${ }^{18}$. The present results showed that internal hip rotational ROM was smaller than external hip rotational ROM, and internal hip rotational ROM in our study was smaller than in a previous study. Elderly adults with severe knee OA may have some limitation in hip ROM, regarded as knee-hipspine syndrome, although hip OA patients were excluded from our study ${ }^{19}$.

This study had limitations that warrant mention. First, the number of patients enrolled was small, which limited the power of the statistical analysis. Second, there was variation in the intraoperative joint gaps. Third, this study only included patients with varus knee alignments. Although most patients with knee OA cases exhibit varus knee deformity, patients with valgus knee deformity should also be evaluated.

Although the hip joint is left intact during TKA, TKA affects hip rotational movement along with correction of alignment and rotation of the knee joint. Therefore, TKA improves knee kinematics and affects hip rotational movement.

\section{Conclusion}

TKA improved both knee function and hip rotational ROM.

Conflict of Interest: The authors declare no conflicts of interest.

\section{References}

1. Muraki S, Oka H, Akune T, et al. Prevalence of radiographic knee osteoarthritis and its association with knee pain in the elderly of Japanese population-based cohorts: the ROAD study. Osteoarthritis Cartilage. 2009;17:113743.

2. Alkan BM, Fidan F, Tosun A, Ardicoglu O. Quality of life and self-reported disability in patients with knee osteoarhtirits. Mod Rheumatol. 2014;23:166-71.

3. Fukutani N, Iijima H, Aoyama $\mathrm{T}$, et al. Knee pain during activities of daily living and its relationship with physical activity in patients with early and severe knee osteoarthritis. Clin Rheumatol. 2016;35:2307-16.

4. Brandes M, Ringling M, Winter C, Hillmann A, Rosenbaum D. Changes in physical activity and health-related quality of life during the first year after total knee arthroplasty. Arthritis Care Res (Hoboken). 2011;63:328-34.

5. George LK, Rulz D Jr, Sloan FA. The effects of total knee arthroplasty on physical functioning in the older population. Arthritis Rheum. 2008;58:3166-71.

6. Chadayammuri V, Garabekyan T, Bedi A, et al. Passive hip range of motion predicts femoral torsion and acetabular version. J Bone Joint Surg Am. 2016;98:127-34.

7. Han H, Kubo A, Kurosawa K, et al. Ipsilateral patterns of the rotational range of motion of the hip in healthy Japanese adults. J Phys Ther Sci. 2016;28:2250-5.

8. Hauschild O, Muenzberg M, Knothe D, et al. Rotational limb alignment changes following total knee arthroplasty. Knee Surg Sports Traumatol Arthrosc. 2013;21:2346-54.

9. Bellemans J, Colyn W, Vandenneucker H, Victor J. The Chitranjan Ranawat award: is neutral mechanical alignment normal for all patients? The concept of constitutional varus. Clin Orthop Relat Res. 2012;470:45-53.

10. Cooke TD, Sled EA, Scudamore RA. Frontal plane knee alignment: a call for standardized measurement. J Rheumatol. 2007;34:1796-801.

11. Hernandez RJ, Tachdjian MO, Poznanski AK, Dias LS. CT determination of femoral torsion. AJR Am J Roentgenol. 1981;137:97-101.

12. Okazaki K, Miura H, Matsuda S, et al. Asymmetry of me- 
diolateral laxity of the normal knee. J Orthop Sci. 2006;11: 264-6.

13. Iizawa N, Mori A, Majima T, Kawaji H, Matsui S, Takai S. Influence of the medial knee structures on valgus and rotatory stability in total knee arthroplasty. J Arthroplasty. 2016;31:688-93.

14. Trudel G, Zhou J, Uhthoff HK, Laneuville O. Four weeks of mobility after 8 weeks of immobility fails to restore normal motion. Clin Orthop Relat Res. 2008;466:1239-44.

15. Van Arkle RJ, Amis AA, Cobb JP. The capsular ligaments provide more hip rotational restraint than the acetabular labrum and the ligamentum teres. Bone Joint J. 2015;97-B: 484-91.

16. Van Arkle RJ, Amis AA, Jeffers JRT. The envelope of passive motion allowed by the capsular ligaments of the hip. J Biomech. 2015;48:3803-9.

17. Martin HD, Savage A, Braly BA, Palmer IJ, Beall DP, Kelly B. The function of hip capsular ligaments: quantitative report. Arthroscopy. 2008;24:188-95.

18. Han H, Kubo A, Kurosawa K, Maruichi S, Maruyama H. Hip rotation range of motion in sitting and prone posi- tions in healthy Japanese adults. J Phys Ther Sci. 2015;27: 441-5.

19. Oshima $\mathrm{Y}$, Watanabe N, Iizawa N, Majima T, Kawata M, Takai S. Knee-hip-spine syndrome: Improvement in preoperative abnormal posture following total knee arthroplasty. Adv Orthop. 2019;8484938.

(Received, August 13, 2019)

(Accepted, November 26, 2019)

(J-STAGE Advance Publication, December 27, 2019)

Journal of Nippon Medical School has adopted the Creative Commons Attribution-NonCommercial-NoDerivatives 4.0 International License (https://creativecommons.org/licenses/by-nc-nd/4.0/) for this article. The Medical Association of Nippon Medical School remains the copyright holder of all articles. Anyone may download, reuse, copy, reprint, or distribute articles for non-profit purposes under this license, on condition that the authors of the articles are properly credited. 